\title{
The Impact of a Health Promotion Educational Program on Cardiovascular Risk Factors for HIV Infected Women on Antiretroviral Therapy
}

\author{
Samaha Hodges ${ }^{2}$ and Marcia McDonnell Holstad ${ }^{1 *}$ \\ ${ }^{1} D S N$, FNP-BC, FAANP, FAAN, Associate Professor, Nell Hodgson School of Nursing Emory University, Atlanta, GA, USA \\ ${ }^{2} \mathrm{MPH}$, Project Manager Co-Investigator of the U.S. LATTE Arm, American Cancer Society, Atlanta, GA, USA
}

\begin{abstract}
This study investigates the efficacy of a health promotion educational program on improving cardiovascular risk factors of weight, Body Mass Index, and waist to hip ratio in 76 predominately African American HIV-infected women. The health promotion educational program was the control group (that focused on improving self-efficacy for nutrition, exercise, stress reduction and women's health behaviors) of a NIH-funded study. The majority of participants was overweight, obese, or at high risk based on waist hip ratio at the beginning of the study. There were no statistically significant improvements in body mass index or waist hip ratio from pre intervention to up to 9 months post intervention. There were significant changes in waist hip ratio and body mass index in both directions (improvement and worsened) for a small group of participants. The health promotion program did not affect significant changes in cardiovascular risk and should be revised, lengthened, and refocused on nutrition, diet, exercise, and long term goal commitments to reduce the high risk for cardiovascular disease in this group.
\end{abstract}

Keywords: HIV; Cardiovascular risk; Antiretroviral therapy; HIV infected women

Abbreviations: AA: African American; ART: Antiretroviral Therapy; BMI: Body Mass Index; CVD: Cardiovascular disease; HPP: Health Promotion Educational Program; WHR: Waist Hip Ratio

\section{Introduction}

Antiretroviral Therapy (ART) has significantly lengthened the lives of HIV-infected persons; however, with increased life spans, HIV Associated Non-AIDS Conditions (HANA) such as Cardiovascular Disease (CVD) are emerging as significant causes of morbidity. Reports suggest that the increasing rates of CVD in HIV infected persons may have a relationship to traditional risk factors such as longer life-spans, increased smoking rates, a weakened glucose tolerance and resistance to insulin and dyslipidemia. Other factors such as body composition changes (loss of subcutaneous fat and/or the addition of visceral fat in some HIV positive patients), inflammation, and the direct effects that the virus has on the vasculature in addition to the specific effects of the antiretroviral drugs can also be linked to CVD [1]. Metabolic complications, such as an increased Waist-to-Hip Ratio (WHR) and altered fat distribution are common in HIV infected adults who are receiving ART [1]. Weight gain, a risk factor for CVD, is one of the most troubling ART side effects for women [2].

African American (AA) women account for $11 \%$ of the US population, however they make up $64 \%$ of new HIV infection in women in 2010 indicating a significant disparity [3]. AA women are also disproportionately represented in cases of CVD [4] and as they age are also at risk for morbidity and mortality due to CVD.

This research study examined the efficacy of a Health Promotion Program (HPP) educational intervention on weight reduction and anthropomorphic measures in HIV-infected women on ART. The HPP program consisted of eight group sessions that focused on increasing self-efficacy for nutrition, exercise, stress management, and women's health. This program was specially tailored for HIV-infected women on ART. Specifically we examined the effects of the intervention on weight, hip and waist circumferences, and waist to hip ratios in a subset of the women who took part in this program. The HPP was the control condition of a large randomized trial to examine the efficacy of a motivational group intervention program to promote ART adherence and use of risk reduction behaviors in HIV-infected women [5].

\section{Background}

There is substantial evidence that ART is saving lives, however there are risks that cannot be ignored: one such risk is that which results from ART and HIV-infection alone and in combination on an increase prevalence for CVD. The role of obesity in predisposing individuals to CVD risk is well established. Waist circumference, abdominal girth, WHR and BMI are all useful indicators of obesity.

\section{Obesity as a CVD risk factor}

Abdominal obesity as well as overall obesity has been linked with CVD risk in both women and men [6]. Having a high waist circumference as an HIV infected person can also have an association with having an increased risk for hypertension, type 2 diabetes, dyslipidemia, and CVD in persons with a BMI that falls within a range between 25 and $34.9 \mathrm{~kg} / \mathrm{m}^{2}$. In those patients that may fall into the obese category, metabolic complications or changes in waist circumference are useful predictors of changes in risk factors for CVD. In some cases, waist circumference is a better warning sign of relative disease risk than BMI. Waist circumference may be a better indicator for calculating risk for obesity-related disease in older adults [7].

*Corresponding author: Marcia McDonnell Holstad, FNP-BC, FAANP, FAAN, As sociate Professor, Nell Hodgson School of Nursing Emory University, Atlanta, GA, USA; Tel: 404.727.1307; Fax: 404.727.8506; E-mail: nurmmcd@emory.edu

Received May 27, 2013; Accepted July 23, 2013; Published July 27, 2013

Citation: Hodges S, Holstad MM (2013) The Impact of a Health Promotion Educational Program on Cardiovascular Risk Factors for HIV Infected Women on Antiretroviral Therapy. J AIDS Clin Res 4: 224. doi:10.4172/2155-6113.1000224

Copyright: (c) 2013 Holstad MM, et al. This is an open-access article distributed under the terms of the Creative Commons Attribution License, which permits unrestricted use, distribution, and reproduction in any medium, provided the original author and source are credited. 
The Body Mass Index (BMI) is defined as a measure of weight in relation to height. BMI is broadly utilized in classifications for overweight and obesity. CVD risk continually increases as the BMI increases. A BMI of over 25 is considered overweight, while someone who is classified as obese will have a BMI of over 30. Having a BMI as low as 21 , may serve as the greatest protection from CVD death in women [8]. The prevalence of BMI $\geq 30 \mathrm{~kg} / \mathrm{m}^{2}$ is disproportionately higher in many racial and ethnic populations, especially in African American women, Puerto Ricans, Mexican Americans, several American Indian and Native Hawaiians, Pacific Islanders and Alaska Native populations [9].

Research conducted by Hu examined physical activity associations and different indicators of obesity (BMI, WHR, and waist circumference) with the risk of CVD and found that BMI was a better predictor of CVD risk in women [10]. In a Swedish prospective study, evidence suggested that there was a positive association between WHR and incidences of myocardial infarction, stroke and death from all causes in both men and women, after adjusting for BMI [11]. Gregg et al. conducted a study examining secular trends in cardiovascular disease risk factors and compared that to body mass index in US adults. They examined five cross-sectional, nationally representative surveys to determine 40year trends in CVD risk factors by BMI groups among adults' ages 20 to 74 years in the US. The total number of participants in the five surveys equaled 48,853 people. The results of this study showed that although prevalence for risk factors in CVD declined over the 40 year span, people categorized as obese still have higher risk factor levels as compared to a person categorized as lean [12]. In a sample of HIV-infected persons (including $40 \%$ women) increased waist circumference was a significant predictor of CVD risk [13]. In that sample, the mean waist measure for women was $99.3 \mathrm{~cm}(\mathrm{SD}=13.2)$ indicating abdominal obesity and their mean $\mathrm{BMI}$ was $29.0(\mathrm{SD}=5.9)$, indicating overweight. When compared to men who were living with HIV from 1 to 20 years, a significantly greater proportion of HIV infected women had abdominal obesity.

\section{ART as a risk factor}

Researchers compared $21 \mathrm{HIV}$-infected women who communicated that they had serious concerns about changes in body habitus or their physical make-up during their course of ART treatment with 21 women who received ART but did not complain of physical body make-up changes. Both groups received physical examinations and anthropometric measurements; serum lipid and endocrine assays were taken as part of the evaluation. The frequency of changes reported by the 21 symptomatic women were: increase in abdominal size $(90 \%)$, increase in breast size (71\%), weight gain of $>5 \mathrm{~kg}(43 \%)$, peripheral fat wasting $(43 \%)$ and buttock fat wasting (38\%). The comparison group patients reported experiencing an increase in abdominal size (29\%) and weight gain $>5 \mathrm{~kg}$ (19\%), but, out of the group no one reported experiencing clinically detectable peripheral or buttock fat wasting, or increased breast size. Mean waist circumference, WHR, body fat, and BMI were above the desirable range for women in both groups. This study's findings suggest that women receiving ART medication encounter changes in body habitus which is caused by redistribution of fat. The observed changes in body fat distribution are alterations that have been strongly correlated with increased risk for CVD [14].

Additionally, the DAD (Data Collection on Adverse Events of Anti-HIV Drugs) study, a multinational, tri-continental collaboration between ongoing HIV cohort studies was initiated in December 1999. DAD's focus was to try to detect any incidence of stroke and myocardial infarction while attempting to determine whether or not the exposure time to any components within the antiretroviral medication regimen could be exclusively found to be associated with cardiovascular risk. Analysis objectives were to determine the extent of patients that had an elevated risk profile for CVD at the time of inclusion into the DAD study. Additionally, DAD researchers aimed to pinpoint any factors associated with these increased risk profiles, more specifically, factors in regards to the type and duration of ART therapy. Authors concluded that although the use of these drugs does aid in effective suppression of the HIV virus and builds stronger immunity, the downside is that they are also associated with a high prevalence of multiple risk factors for CVD. Those risks are increased even further for patients that are receiving ART drug therapy from the three classes of drugs being used simultaneously as standard care at the time of the study [15].

\section{Interventions to reduce CVD risk in women}

The North Carolina WISEWOMAN project was initiated as an intervention for low-income women in the state of North Carolina to evaluate the ease of expanding a current health screening program to include CVD screening and interventions. This project was a nonrandomized group assigned intervention trial that contained two treatment groups: Minimum Intervention (MI) and Enhanced Intervention (EI). WISEWOMAN looked to expound upon a program that already existed to include a new aspect; which was the addition of a CVD screening program that contained a diet and exercise intervention in order to reduce CVD risk in the women that participated. Seventeen North Carolina county health departments were designated as MI centers and 14 were EI centers. The EI centers included three specifically designed counseling sessions over a course of 6 months using assessments and programs tailored to women with low incomes. At the conclusion of the study the total Dietary Risk Assessment (DRA) score improved (decreased) by 2.1 units in the EI group and remained unchanged in the MI group. The improvement in dietary risk score among the EI groups was statistically significant $(p=0.001)$. CVD risk factor levels improved slightly over time in both EI and MI groups. The percentage of women that reported physical activity increased for both EI (5.9\%) and MI (9.3\%), however was not statistically significant [16].

The WISEWOMAN Project was also conducted in Massachusetts. The results of this study showed a 7\% reduction in hypertension among EI site women $(p=0.02)$. The occurrence of a reduction is an important factor because hypertension is a risk factor for CVD. Eighteen percent more women in the EI site group were more physically active than the $6 \%$ in the MI site group [17]

To improve physical activity for women in small rural communities (populations of less than 25,000), the Physical Activity Intervention for Women was initiated. To avoid cross-intervention contamination that could threaten study validity, community randomization was done rather than by participant. This randomized community based clinical trial was conducted on 42 women ages $35-65$ years old. The majority of the sample was Caucasian (95\%), married (60\%), employed outside the home $(60 \%)$, and had earned a bachelor's degree or higher in education (75\%). The experimental group received a Heart and Soul Physical Activity Program (HSPAP) intervention consisting of a HSPAP interactive booklet, 12 weekly group meetings, physical activity materials and self-esteem support. The comparison group received the AHA booklet, "Exercise and Your Heart" [18] and 1 hour verbal instructions that was a summation of the booklet, physical activity instructions and instructional video. In addition the comparison group also received one follow-up phone call after the initial instructional video. Results of this study showed that between baseline and 12 weeks, the comparison group women increased their physical activity by 67 minutes a week on average. The women in the intervention group 
increased their time by 141 minutes a week on average. By 12 weeks both groups had exceeded 150 minutes a week, which is the minimum amount recommended by Healthy People 2010 [18]. We could find no CVD risk reduction interventions specifically targeted for HIV-infected women.

\section{Research Question}

Will there be a significant decrease in the anthropometric measurements (BMI, weight, waist, hip circumference, and WHR) of HIV-infected women on ART after attending the 8 session HPP from pre-HPP to post intervention follow-up points?

\section{Methods}

\section{Design}

This project is a secondary analysis of data collected from the KHARMA (Keeping Healthy and Active with Risk Reduction and Medication Adherence) Project, a Randomized Controlled Clinical Trial (RCT) that tested the efficacy of a nurse-led motivational group intervention to promote adherence to ART and risk reduction behaviors in HIV-infected women compared to an attention equivalent HPP control group led by nurses and a health educator $[5,19]$. We wanted to find out if the control HPP group had any effect in reducing CVD risk factors of weight, BMI, and WHR.

The study was approved by the Emory University IRB and the Research Committee at the primary site. Data collection occurred between 2005 and 2008. All participants completed a baseline assessment where they were randomized to either the intervention or control (HPP) condition. Groups started about two weeks after baseline. Follow-up assessments for all participants were completed immediately (2 weeks) and 3, 6, and 9 months after the final group session in each condition. Weight, waist and hip measures were taken from only the HPP participants at group session 1 (pre-intervention) and session 8 and again at 2 weeks and 9 months post intervention. For this project height data was extracted from participant medical records at the primary research site after completion of the RCT.

\section{The HPP intervention}

The HPP was the attention equivalent control condition of The KHARMA Project. It consisted of eight weekly sessions lasting from 1.5 to 2 hours in length with six to eight women per group. The sessions were led by a nurse and a health educator and focused on nutrition, exercise, stress reduction and women's health topics. All content was tailored to the needs of HIV-infected women on ART and each participant received a workbook that contained all the program content, including supplementary materials. There was an introductory session that provided an overview of the program and introduction of individual group members and group termination occurred in the last session. Educational strategies for the program included lecturediscussion and educational games and other learning activities. The HPP topics are listed in Table 1. The first five of the eight sessions focused on cardiovascular risk reduction: an introductory overview (first session), nutrition (two sessions), physical activity and exercise (two sessions). The nutrition session's content included healthy food choices (for energy and immune function), portion size and food safety, information about cholesterol and fat and reading food labels. Physical activity and exercise sessions discussed information on physical changes associated with HIV and ART, weight change, and maintaining weight. Content stressed the importance of being physically active and reviewed how to increase physical activity into one's daily routine.
Introduction \& Overview of the effects of nutrition, exercise and stress on the body and immune system

Nutrition Part I: Eating for Energy

Nutrition Part II: Cholesterol, Fat, \& Label Reading

Exercise \& Fitness Part I: The Awareness of Physical changes and how to deal with them

Exercise \& Fitness Part II: The Importance of a Physically Active Lifestyle

Weight Changes: Recognizing the ups and downs of body weight

Stress \& Depression: Signs, Symptoms, \& Some Solutions

General Wellness: Lung cancer and smoking cessation and Lymphoma

Women's Health: The Importance of Breast Self-exam and Understanding Menstruation \& Menopause

Women's Health: Recognizing \& Understanding Gynecological Problems

Note: Reproduced from OJIN: The Online Journal of Issues in Nursing (used with permission)

Table 1: Topical Outline Health Promotion Program by Group Session.

Women learned simple exercises they could perform at home to tone and strengthen muscles.

The theoretical framework for the HPP was Social Cognitive Theory [20]. The program was designed to increase self-efficacy and personal goal setting related to the topics described above.

\section{Setting and sample}

To participate in KHARMA, women met the following eligibility criteria: a) female by birth; b) infected with HIV; c) prescribed antiretroviral medications; d) 18 years of age or older; e) English speaking; f) mentally stable as determined by a screening assessment; and g) willing to participate by completing five computerized assessments, use electronic drug monitoring caps, be randomly assigned and participate in either the Health Promotion or the Motivational Group.

Women were recruited from five HIV care sites in a large metropolitan Southeastern city. The primary site was a large infectious disease clinic that served over 4000 HIV-infected men, women, and children. Eligibility was assessed in person by KHARMA project staff. Potential participants would give KHARMA referral forms (which verified eligibility) signed by their provider to staff. Staff would then administer a screening assessment that tested potential participants for cognitive impairment and severe depressive symptoms and suicidal tendencies. Participants who had cognitive impairment that was determined to be severe were not eligible for the study. Those whose results showed severe depressive symptoms and/or suicidal ideations were immediately referred to mental health services and were able to be rescreened after receiving mental health care, usually in 2-3 weeks.

A total of 249 women were screened for the KHARMA Project, 207 (83\%) of whom were eligible and completed baseline interviews. A total of 103 women were randomized to the HPP condition and 19 HPP groups were held over the study period. The sample for the current study was limited to 76 of the 103 participants from the HPP who received care at the primary site and had height data available in their medical record (needed for BMI calculation). The sample is smaller because height data was not available in all primary site participants' medical records.

\section{Measures}

Measurements of weight, waist, and hips were taken and recorded on a data record form for each participant. The same tape measure and scale were used for all participants. The scale used in the study was calibrated regularly. KHARMA project staff were trained in how to take all measurements and inter rater reliability was calculated for 
waist and hip measures within $0.5 \mathrm{~cm}$ agreement between staff on two 'standardized' women prior to initiating the study participant measurements. BMI was calculated from height measurements obtained from medical records and recorded weights. The formula used for BMI calculations was derived from the standard imperial BMI formula (BMI $\mathrm{kg} / \mathrm{m}^{2}=$ weight in pounds ${ }^{*} 703 /$ height in inches ${ }^{2}$ ) [21]. The formula used to calculate WHR was derived from standard waist to hip ratio formula (dividing waist circumference in inches/hip circumference in inches) [22]

\section{Data analysis}

Data on the 76 HPP participants were entered into a database and SPSS statistical software version 17 was used for analyses. A one-way ANOVA test, a paired samples $t$, Pearson's $r$ correlations, and a Chi Square analysis of WHR and BMI measurements were conducted. Descriptive statistics including means, standard deviations, frequencies, and crosstabulations were calculated to describe the sample. Significance levels were set at $p=.05$.

\section{Missing data}

The sample size for the analysis for BMI and WHR was reduced due to missing data from participant's failure to attend a group session, a follow-up interview, or by having incomplete medical records (i.e., no height recorded). For these analyses only existing data was analyzed and numbers of participants $(n)$ are reported for each analysis. We chose not to impute missing data. Because of the large numbers of missing data we felt it might misrepresent the sample $(47.4 \%[n=36]$ of the participants lacked WHR and $61.8 \%$ [ $n=47$ ] lacked BMI data).

\section{Results}

\section{Sample}

Of the 76 participants 74 (97.4\%) were African American. The average age at baseline was 44.5 years with a range of 23 to 68 years old. A description of the full sample is presented elsewhere [5].

\section{Weight}

We used national standard criteria set by the Centers for Disease Control to categorize women by BMI into underweight $(<18.5)$, normal weight (18.5 - 24.9), overweight (25.0-29.9) and obese ( $\geq 30.0)$ [23]. To categorize women based on the WHR we used the WHR calculator criteria, where a ratio of $<0.8$ is low risk, 0.81 to 0.85 is moderate risk and $\geq 0.85$ is high risk [22]. Frequency and percentages of women in each category pre- and post-intervention can be found in Tables 2, 3, and 4 . Of the 76 women in the data set, $57(75 \%)$ had a weight change

\begin{tabular}{|c|c|c|c|c|c|}
\hline Pre-Intervention & Frequency & Percent & Post Intervention & Frequency & Percent \\
\hline Normal Weight & 8 & $27.6 \%$ & Normal Weight & 9 & $31.0 \%$ \\
\hline Overweight & 12 & $41.4 \%$ & Overweight & 4 & $13.8 \%$ \\
\hline Obese & 9 & $31.0 \%$ & Obese & 16 & $55.2 \%$ \\
\hline Total & $\mathbf{2 9}$ & $\mathbf{1 0 0}$ & Total & $\mathbf{2 9}$ & $\mathbf{1 0 0}$ \\
\hline
\end{tabular}

Table 2: BMI Categories Pre and Post Intervention Using National Standards $(n=29)$

\begin{tabular}{|c|c|c|c|c|c|}
\hline Pre-Intervention & Frequency & Percent & Post Intervention & Frequency & Percent \\
\hline Low Risk & 2 & $5.0 \%$ & Low Risk & 2 & $5.0 \%$ \\
\hline Moderate Risk & 3 & $7.5 \%$ & Moderate Risk & 6 & $15.0 \%$ \\
\hline High Risk & 35 & $87.5 \%$ & High Risk & 32 & $80.0 \%$ \\
\hline Total & $\mathbf{4 0}$ & $\mathbf{1 0 0 \%}$ & Total & $\mathbf{4 0}$ & $\mathbf{1 0 0 \%}$ \\
\hline
\end{tabular}

Table 3: WHR Categories Pre and Post Intervention Using National Standards ( $\mathrm{n}$ $=40$ ).

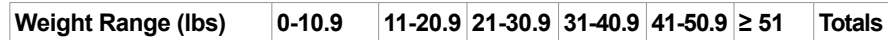
\begin{tabular}{|l|l|l|l|l|l|l|} 
Number (\%) who Gained $18(69 \%)$ & $5(19 \%)$ & $1(4 \%)$ & $1(4 \%)$ & $1(4 \%)$ & 0 & 26
\end{tabular} \begin{tabular}{|l|l|l|l|l|l|l|}
\hline Number who Lost & $22(71 \%)$ & $2(6 \%)$ & $4(13 \%)$ & $1(3 \%)$ & 0 & $2(6 \%)$ \\
31
\end{tabular}

Table 4: Number (and Percent) of Participants Who Gained or Lost Weight in Pounds by the End of the Study $(n=57)$.

at the completion of the program. Of those $57,54 \%(n=31)$ lost weight and $46 \%(n=26)$ gained weight. The average weight lost was 9.98 pounds. The average weight gain was 12.4 pounds.

\section{BMI and WHR}

BMI: A paired-sample t-test was calculated to compare the mean BMI measurements pre-intervention (group session 1) to the BMI measurements post intervention (furthest follow-up time point with data available) in 29 participants with available data. The mean BMI for the pre-intervention was $29.32(S D=7.39)$ and the mean BMI for the post intervention was $29.99(S D=8.04)$. There was a non-significant increase in BMI values from pre to post intervention $(t(28)=-1.141$, $p=.13$ ). With BMI means of 29.32 and 29.99 respectively, participants fall into the overweight category at all time points. Mean BMI values increased from pre-intervention to post intervention by 0.67 .

A one-way repeated measures ANOVA was calculated on 11 participants (who had data for all time points) comparing BMI's at all four time points: group session 1, group session 8 , follow-up 1 (2 weeks) and follow-up 4 (9 months). No significant differences in means at each time point was found $(F(3,30)=1.421, p=.266)$ : the means for group session $1(m=31.07 S D=9.190)$, group session $8(m=32.18$, $S D=9.475)$, follow-up $1(m=32.27, S D=9.952)$ and follow-up $4(m=$ $32.08, S D=9.685)$. Mean BMI values during all four time points were in the obese category being $\geq 30$. Based on Pearson correlations, there was no significant correlation between age and BMI, however, there was a strong positive correlation between pre-intervention and post intervention BMI $(r(20)=.876, p=.000)$. Participants' BMI increased from pre-intervention to post intervention.

WHR: Similar to BMI, a paired-samples $t$-test was calculated to compare the mean WHR measurements pre-intervention time point to the WHR measurements post intervention time point on 40 participants who had data available. The means for the pre and postintervention did not change ( $p=0.24): 0.91(S D=0.066), 0.91(S D=$ $0.063)$ respectively.

A one-way repeated measures ANOVA was calculated on 24 participants with data for all time points comparing WHR's at each of the four different time points with again, no significant changes found $(F(3,69)=0.454, p=0.715)$. Calculated means at each time point $(>$ 0.85 ) were in the high risk category. Similar to BMI, Pearson correlation showed no significant relationship between participant's age and WHR. A weak and significant correlation between pre-intervention and post intervention WHR was found $(r=(39)=0.335, p=0.016)$.

In order to determine if there were changes in risk category for the individual participants' WHR and BMI before and after the intervention, we conducted Chi square analyses to compare those categorized at group 1 vs. post-intervention (the furthest time point data was available for a participant). These time points were chosen because they contained the most available data. For BMI: eight participants remained in the normal weight category, one participant moved from overweight to normal weight, four remained in the overweight category, seven moved from overweight to obese category, and nine remained in the obese category $(n=29, p<0.001)$. For WHR: one participant stayed in the low risk category, one moved from low to high risk, three moved from high into 
the moderate risk category and 31 remained in the high risk category $(n=40, p<0.001)$.

\section{Discussion}

We conducted a secondary analysis to determine the effects of a health promotion group intervention on outcomes of weight, BMI and WHR in $76 \mathrm{HIV}$-infected women who were prescribed antiretroviral therapy. The HPP was the control condition of a large behavioral clinical trial focused on promoting adherence to ART and use of risk reduction behaviors. Over the 9-month follow-up period, we found no significant decrease in anthropometric measurements in the HIV-infected women that attended the eight-session HPP. For each time point the majority of participants were classified as high risk category for WHR and obese category for BMI.

While examining the data, it was clear that attendance in group sessions and for follow-up assessments for this sample dropped by the time of the 9 month final follow-up. This attrition affected the sample size in subsequent analyses.

Mean WHR did not change over time: four participants improved their status, one worsened her status, and the remainder stayed the same. BMI values had a non-statistically significant mean increase of 0.67: eight women maintained normal weight, one improved to normal, seven participants worsened their status, and nine remained obese. There was a correlation between pre and post intervention values for both WHR and BMI.

The degree of cardiovascular risk based on WHR and BMI noted in this small middle aged sample of women on ART is a concern and warrants further investigation and more focused intervention. Anecdotally, these results are not surprising. During the group sessions women were provided healthy snack options. These items included fresh fruit, water, fruit juices, sandwiches and other healthy options. The women objected to these choices and lobbied strongly to have fried chicken, pizza and sodas. This demonstrates that changing one's life-long eating behavior requires additional culturally appropriate strategies and possibly more than an eight week time commitment.

The HPP was provided as an educational attention equivalent control condition and although it was based on strong framework of self-efficacy and sound evidence, it was very broad in focus and not designed to be a rigorous and focused program to reduce cardiovascular risk. The 'dose' as well as strategies related to weight loss, exercise and nutrition may not have been strong enough to promote behavior change. Also, it was not the primary focus of the project and consequently may have engendered lesser interest on the part of the researchers and participants. Longer and more focused interventions, such as the WISEWOMAN [17] and Physical Activity Intervention for Women [18] have been effective however these programs were not targeted to HIV-infected women. Fitch et al. demonstrated that lifestyle modification was sufficient to improve the intake of calories, saturated fats and fiber along with increasing physical activity improved waist circumference and blood pressure in HIV positive patients with metabolic syndrome [24].

Everyone that participated in the KHARMA Project was on ART, some participants were newer to medication than others, however the average time on ART was 6.8 years for the larger HPP control sample $(n=102)$ and 6.1 years for the entire KHARMA sample. ART has been associated with side effects of body habitus changes such as lipodystrophy and increased abdominal girth [25]. Abdominal obesity was more prevalent in women compared to men with HIV diagnosis from 1 to 20 years [13]. The average duration of HIV in the HPP sample was 10.5 years.

This study had several limitations. The small sample size and predominance of African Americans can reduce the study's potential for generalizability to the broader population of HIV-infected women, though in the US, AA represent a disproportionate number of HIVinfected women. Other limitations included missing measurements data due to the women not being present for all of their scheduled groups and follow-ups and lack of height information in participant medical records. Measurement inconsistencies are another limitation. Although the same instruments were used throughout the study, error in scale calibration could have occurred. Additionally, participants clothing could have been an interference when measuring waist and hip circumference. Also, measurements that were taken on participants during their group sessions were not necessarily done by the same staff person for follow-up sessions.

\section{Conclusion}

In the US, obesity, a risk factor for CVD, is continuously increasing among the general population. Our data reflect a high level of obesity and high risk WHR in this sample of predominately African American HIV-infected women.

The HPP was a comprehensive eight session health education program targeted for HIV-infected women. It had multiple components, such as nutrition, exercise, women's health, stress reduction, and was implemented as an attention equivalent control condition. There were no significant improvements in anthropomorphic measures in HIV-infected women on ART who were randomized to that group. It should be revised and refocused to a more intense and targeted long term intervention, specifically tailored to the target group. Additions to the program should also address the issues of weight regain (relapse), accessibility to exercise equipment and nutritious healthy and culturally appropriate foods. Strategies such as motivational interviewing, which was a used in the KHARMA intervention to successfully promote adherence and use of some risk reduction behaviors [5] have also shown promise for healthy eating [26].

In the United States, ethnic minorities experience higher rates of obesity than do their white counterparts. Accompanying these higher rates of obesity is an increased prevalence of obesity-related diseases, such as CVD. Lifestyle and other factors can contribute negatively on the types of food that is purchased and consumed by this population. Circumstances such as these underscore the importance of finding effective weight management strategies to use with these groups. A potential resolution could be the use of lifestyle behavioral programs that focus on making gradual changes over time may offer the most promise for improvement, as short-term interventions have not been beneficial for weight loss with minority groups [27].

\section{Acknowledgement}

ROI NR008094 National Institute of Health (NIH)/ National Institute of Nursing Research and in part by Emory Center for AIDS Research (P30 AI050409)

\section{References}

1. Grinspoon SK, Grunfeld C, Kotler DP, Currier JS, Lundgren JD, et al. (2008) State of the science conference: Initiative to decrease cardiovascular risk and increase quality of care for patients living with HIVIAIDS: executive summary. Circulation 118: 198-210.

2. Holstad MM (2007) Antiretroviral side effects and their distress in HIV+ Women in Association of Nurses in AIDS Care (ANAC). Annual Meeting Abstracts.

3. Centers for Disease Control and Prevention (2013) HIV Among Women. CDC Fact. 
Citation: Hodges S, Holstad MM (2013) The Impact of a Health Promotion Educational Program on Cardiovascular Risk Factors for HIV Infected Women on Antiretroviral Therapy. J AIDS Clin Res 4: 224. doi:10.4172/2155-6113.1000224

Page 6 of 6

4. Daroszewski EB (2004) Dietary fat consumption, readiness to change, and ethnocultural association in midlife African American women. J Community Health Nurs 21: 63-75.

5. Holstad MM, Dilorio C, Kelley ME, Resnicow K, Sharma S (2011) Group motivational interviewing to promote adherence to antiretroviral medications and risk reduction behaviors in HIV infected women. AIDS Behav 15: 885-896.

6. Rexrode KM, Carey VJ, Hennekens CH, Walters EE, Colditz GA, et al. (1998) Abdominal adiposity and coronary heart disease in women. JAMA 280: 18431848.

7. NHLBI (2009) Guidelines on Overweight and Obesity: Electronic Textbook, $\mathrm{NIH}$.

8. WHO (2009) Risk Factor: Obesity. Cardiovascular Disease

9. Smith SC, Clark LT, Cooper RS, Daniels SR, Kumanyika SK, et al. (2005) Discovering the Full Spectrum of Cardiovascular Disease: Minority Health Summit 2003: Report of the Obesity, Metabolic Syndrome, and Hypertension Writing Group. AHA Conference Proceedings, Circulation111: e134-e139.

10. Hu G, Tuomilehto J, Silventoinen K, Barengo N, Jousilahti P (2004) Joint effects of physical activity, body mass index, waist circumference and waist-to-hip ratio with the risk of cardiovascular disease among middle-aged Finnish men and women. Eur Heart J 25: 2212-2219.

11. Wing RR, Matthews KA, Kuller LH, Meilahn EN, Plantinga $P$ (1991) Waist to hip ratio in middle-aged women. Associations with behavioral and psychosocia factors and with changes in cardiovascular risk factors. Arterioscler Thromb 11 1250-1257.

12. Gregg EW, Cheng YJ, Cadwell BL, Imperatore G, Williams DE, et al. (2005) Secular trends in cardiovascular disease risk factors according to body mass index in US adults. JAMA 293: 1868-1874.

13. Capili B, Anastasi JK, Ogedegbe O (2011) HIV and general cardiovascular risk. J Assoc Nurses AIDS Care 22: 362-375.

14. Dong KL, Bausserman LL, Flynn MM, Dickinson BP, Flanigan TP, et al. (1999) Changes in body habitus and serum lipid abnormalities in HIV-positive women on highly active antiretroviral therapy (HAART). J Acquir Immune Defic Syndr 21: 107-113.

15. Friis-Møller N, Weber R, Reiss P, Thiébaut R, Kirk O, et al. (2003) Cardiovascular disease risk factors in HIV patients--association with antiretroviral therapy. Results from the DAD study. AIDS 17: 1179-1193.

16. Rosamond WD, Ammerman AS, Holliday JL, Tawney KW, Hunt KJ, et al. (2000) Cardiovascular disease risk factor intervention in low-income women: the North Carolina WISEWOMAN project. Prev Med 31: 370-379.

17. Stoddard AM, Palombo R, Troped PJ, Sorensen G, Will JC (2004) Cardiovascular disease risk reduction: the Massachusetts WISEWOMAN project. J Womens Health (Larchmt) 13: 539-546.

18. Peterson JA, Yates BC, Atwood JR, Hertzog M (2005) Effects of a physical activity intervention for women. West J Nurs Res 27: 93-110.

19. Holstad MM, Dilorio C, Magowe MK (2006) Motivating HIV positive women to adhere to antiretroviral therapy and risk reduction behavior: the KHARMA Project. Online J Issues Nurs 11: 5

20. Bandura $A$ (1986) The social foundation of thought and action: A social cognitive theory. Prentice Hall, NJ.

21. DHHS (2009) Body Mass Index. National Institute of Health-Obesity Education Initiative.

22. BMI-WHR (2009) BMI Calculator: Waist to Hip Ratio calculator

23. CDC (2009) BMI for adults. National Center for Chronic Disease, Prevention \& Health Promotion.

24. Fitch KV, Anderson EJ, Hubbard JL, Carpenter SJ, Waddell WR, et al. (2006) Effects of a lifestyle modification program in HIV-infected patients with the metabolic syndrome. AIDS 20: 1843-1850.

25. Lichtenstein K, Balasubramanyam A, Sekhar R, Freedland E (2007) HIV associated adipose redistribution syndrome (HARS): definition, epidemiology and clinical impact. AIDS Res Ther 4: 16.

26. Resnicow K, Wallace DC, Jackson A, Digirolamo A, Odom E, et al. (2000) Dietary change through African American churches: baseline results and program description of the eat for life trial. J Cancer Educ 15: 156-163.

27. Tyler C, Craig AJ, Foreyt JP (2007) Themed Review: Lifestyle Management of Obesity. Am J Lifestyle Med 1: 423-429. 